\title{
Prácticas pedagógicas innovadoras mediadas por las TIC
}

\author{
LINA PARRA BERNAL ${ }^{*}$ \\ Universidad Católica de Manizales (UCM) - Colombia
}

KAREN RENGIFO RODRÍGUEZ ${ }^{* *}$

Servicio Nacional de Aprendizaje (SENA)- Colombia

Recibido el 21-10-20; primera evaluación el 19-07-21;

segunda evaluación el 22-08-21; aceptado el 25-08-21

\section{RESUMEN}

Este artículo presenta el análisis de las prácticas pedagógicas innovadoras mediadas por TIC, realizadas por los profesores de una institución educativa en Popayán Cauca, (Colombia). La investigación es cualitativa, de diseńo biográfico-narrativo, con alcance descriptivo y desarrollada a través de entrevistas semiestructuradas aplicadas a tres profesores. Los resultados permiten establecer que los profesores aplican innovación incremental dentro del aula de clase, en función de las necesidades curriculares y la praxis cotidiana, pero no utilizan continuamente herramientas Web 2.0 y Entornos Virtuales de Aprendizaje, para articular el trabajo extra clase; entre las razones de esta situación se tienen los problemas de conectividad, la resistencia al cambio y la escasa gestión educativa institucional.

Palabras clave: innovación, innovación educativa, práctica pedagógica, TIC.

\section{Innovative ICT-based Pedagogical Practices}

\section{Abstract}

This article presents the analysis of the innovative pedagogical practices mediated by ICT, carried out by the teachers of an educational institution in Popayán Cauca, (Colombia). The research is qualitative, with a biographical - narrative design, with a descriptive scope and developed through semi-structured interview applied to three teachers. The results allow establishing that teachers apply

\footnotetext{
* Doctora en Educación con Especialidad en Mediación Pedagógica por la Universidad de La Salle, Costa Rica. Docente investigadora en la Facultad de Educación de la Universidad Católica de Manizales. Línea de investigación: Educación, Innovación y TIC. https://orcid.org/0000-0001-9838-4783. Correo electrónico: lrparra@ucm.edu.co

** Magíster en Gestión del Conocimiento Educativo por la Universidad Católica de Manizales. Docente Servicio Nacional de Aprendizaje - SENA. Psicóloga. Especialista en Entornos Virtuales de Aprendizaje. https://orcid.org/0000-0002-3189-6853. Correo electrónico: karenr227@hotmail.com
} 
incremental innovation within the classroom, depending on the curricular needs and daily practice, buy they do not continuously use Web 2.0 tools and Virtual Learning Environments, to articulate extra-class work; among the reason from this situation there are connectivity problems, resistance to change and poor institutional educational management.

Keywords: innovation, educational innovation, pedagogical practice, ICT.

\section{Práticas pedagógicas inovadoras mediadas por TIC}

\section{RESUMO}

Este artigo apresenta a análise de práticas pedagógicas inovadoras mediadas por TIC, realizada por professores de uma instituição de ensino em Popayán - Cauca, (Colômbia). A pesquisa é qualitativa, de desenho biográfico-narrativo, com escopo descritivo e desenvolvida por meio de entrevistas semiestruturadas aplicadas a três professores. Os resultados possibilitam estabelecer que os professores aplicam inovação incremental dentro da sala de aula, com base nas necessidades curriculares e na prática cotidiana, mas não utilizam continuamente ferramentas da Web $2.0 \mathrm{e}$ Ambientes virtuais de Aprendizagem, para articular trabalhos extraclasses; as razóes para esta situaçáo incluem problemas de conectividade, resistência à mudança e má gestão educacional institucional

Palavras-chave: inovação, inovação educacional, prática pedagógica, TIC.

\section{INTRODUCCIÓN}

Las tecnologías de la información y la comunicación (TIC), dentro de una sociedad global con nuevos retos y posibilidades en el campo educativo, pueden utilizarse de forma positiva para transformar las prácticas pedagógicas dentro y fuera del aula, potenciando el aprendizaje a través de estrategias colaborativas, creativas, innovadoras y significativas. Con el advenimiento de la era digital y una nueva forma de producir conocimiento, aplicarlo y gestionarlo, las TIC se han convertido en fundamentales para transformar el sistema educativo, donde el desarrollo de habilidades y competencias digitales son vitales para desempeñarse competitivamente. Así, la internet ha pasado de ser un soporte de información o de suministro de contenidos a los usuarios, para convertirse en un soporte tecnológico donde los usuarios crean los contenidos y utilizan las herramientas en línea para hacer su trabajo, como lo evidencia la aparición de las redes sociales, los blogs, los LMS (sistemas de gestión del aprendizaje) como la plataforma Edmodo o Google Classroom, los RED (recursos educativos distribuidos) y las diferentes plataformas virtuales que ofrecen herramientas educativas. 
En este aspecto, la educación no ha estado alejada de los beneficios que ofrecen estas herramientas y es por esto que hoy encontramos prácticas pedagógicas mediadas por las TIC, que centran su propósito en implementar nuevas estrategias, que flexibilicen los aprendizajes y permitan irse desligando de los modelos tradicionales que hoy en día, aun se pueden ver reflejados en el aula de clase. Salinas (2008) afirma que "las instituciones educativas deben revisar sus referentes actuales y promover experiencias innovadoras en el campo de los procesos de enseñanza-aprendizaje apoyados en las TIC» (p. 19). De acuerdo a lo anterior, la institución educativa que participa del estudio en aras de ofrecer una educación de calidad se ha propuesto ejercer una formación integral e innovadora en la utilización de las TIC, que favorezca la capacidad crítica, reflexiva y analítica del estudiante, orientándose siempre a mejorar su calidad de vida y a participar en la búsqueda de alternativas de solución a los problemas de su contexto. Desde esta perspectiva, la investigación busca conocer y describir las prácticas pedagógicas de algunos profesores, para reconocer sí en ellas existen rasgos de innovación.

$\mathrm{Al}$ respecto Rivas (2017) plantea:

La innovación educativa es como una fuerza vital, presente en escuelas, educadores, proyectos y políticas, que es capaz de reconocer las limitaciones de la matriz educativa tradicional y alterarla para el beneficio de los derechos de aprendizaje del siglo XXI de nuestros alumnos. (p. 20)

Por ello, el rol del profesor dentro del proceso innovador es fundamental, como lo plantean Pila, Andagoya y Fuertes (2020), al referirse al profesor como el gestor de la innovación y uno de los principales actores dentro de los cambios significativos en el proceso educativo. Porque de su interés y motivación por el cambio en su práctica, surgen iniciativas novedosas que contribuyen al desarrollo de los aprendizajes de los estudiantes y que para el caso de la investigación articulan las TIC en sus contextos. Unesco (2018a, 2018b), dentro de la agenda global de la educación 2030, contempla las destrezas requeridas para un mundo interconectado, las cuales fueron determinadas por más de 750 expertos y de más de sesenta países, que asistieron a la Conferencia sobre Aprendizaje Móvil 2018, examinando «los tipos de destrezas necesarias en y para una economía y sociedad conectada, con un enfoque en las competencias y destrezas digitales» (p. 7). Desde esta perspectiva, se hace relevante empoderar en la construcción de competencias y destrezas digitales a los profesores, de manera que se traduzcan en prácticas innovadoras que mejoren la calidad de los procesos de enseñanza y aprendizaje. 
Otro aspecto relevante que se destaca a partir de la incorporación de las TIC en las prácticas pedagógicas es el referido a las capacidades de los estudiantes para el procesamiento de la información, la creatividad y la innovación, en función de las destrezas asociadas al pensamiento crítico, lógico y la resolución de problemas, entre otros, que han alcanzado los estudiantes como consecuencia de las prácticas innovadoras emprendidas por sus profesores. Desde esta perspectiva, las TIC se convierten en herramientas y medios que fortalecen los procesos de aprendizaje, propician escenarios de innovación en las aulas y se pueden convertir en vehículos para facilitar el interaprendizaje en el que no solo es el profesor el que enseña y el estudiante aprende, sino que el aprendizaje es compartido, en doble vía, favoreciendo vínculos entre el profesor y el estudiante.

\section{MarCo teórico}

\subsection{Innovación educativa}

Las prácticas pedagógicas de los profesores pueden salirse de la rutina tradicional, involucrando estrategias innovadoras que promuevan cambios en los aprendizajes, para Salinas (2004), «la innovación es entendida como la introducción de los cambios que producen mejora, cambios que responden a un proceso planeado, deliberado, sistematizado e intencional» (p. 20), es por esto que asumir la innovación como el proceso de cambio y transformación, le implica al profesor una reflexión continua acerca de su práctica, cuando el profesor reflexiona sobre lo que hace y cómo lo hace, sin duda se preocupa por implementar nuevas estrategias que posibiliten en los estudiantes logros en sus aprendizajes.

Estos cambios con el apoyo de las TIC pueden enriquecer y permitir la generación de nuevos ambientes en los que los estudiantes se convierten en protagonistas de su aprendizaje, las herramientas de la Web 2.0, 3.0 y 4.0 apuestan por la creación de contenidos, el estudiante pasa del rol pasivo de consumidor al de cocreador de sus propias experiencias de aprendizaje. Unesco (2016) plantea que la innovación en educación debe conceptualizarse como una serie de fases de mejoramiento continuo, que empieza con una intencionalidad, seguida de una planificación, de un aprestamiento de recursos, y que desemboca en una fase de implementación y desarrollo, de la que finalmente se llega a la evaluación y balance de resultados, permitiendo entonces la sistematización de la experiencia.

La innovación requiere de un profesor que se movilice entre los ámbitos del pensamiento, la indagación, la reflexión, la creación y la transformación, 
(Parra y Agudelo, 2020, p. 53), por lo tanto, los profesores para enriquecer sus prácticas pueden acudir a procesos innovadores apoyados en las TIC, ya que, de esta manera, propician la generación de nuevo conocimiento, el cual se caracteriza por el uso creativo e innovador de la tecnología, en ambientes en red, colaborativos e interdisciplinarios. A partir de la innovación se consolidan escenarios formativos que propenden por el reconocimiento y apropiación de problemas pertinentes y contextualizados.

Según Carbonell (2001),

La innovación; es una serie de intervenciones, decisiones y procesos, con cierto grado de intencionalidad y sistematización, que tratan de modificar actitudes, ideas, culturas, contenidos, modelos y prácticas pedagógicas. Y, a su vez, de introducir en una línea renovadora, nuevos proyectos y programas, materiales curriculares, estrategias de enseñanza y aprendizaje, modelos didácticos y otra forma de organizar y gestionar el currículum, el centro y la dinámica del aula (p. 17).

Por lo tanto, la innovación implica la generación de una capacidad humana para transformar, crear y recrear el mundo, produciendo transformaciones culturales, construyendo sujetos sociales e históricos que produzcan un cambio profundo en la realidad, con visión crítica. Transformaciones que contribuyen al mejoramiento de los procesos de enseńanza y aprendizaje, al posibilitar la incorporación de nuevas destrezas y competencias en los profesores, quienes pueden diseñar prácticas pedagógicas innovadoras (Unesco, 2016; Carballo, 2006; Fullan y Stiegelbauer, 1991).

De lo anterior, se puede reconocer que la innovación constituye un concepto profundo, que a su vez implica muchos procesos para ser aplicada, favoreciendo la capacidad de transformación de diversas situaciones en las que convive el ser humano. Para innovar se requiere que lo profesores desarrollen nuevas destrezas, comportamientos y prácticas en asociación con el cambio y la adquisición de nuevas creencias y concepciones. En el contexto educativo la innovación implica la renovación pedagógica, los cambios en las praxis de los profesores dentro del aula, las transformaciones en las políticas de gestión educativa; es decir que la innovación educativa es una ruptura radical con los esquemas y cultura existente dentro de la organización educativa, tanto a nivel administrativo y estratégico, como en lo pedagógico. Se trata entonces de un cambio interno a nivel de las partes interesadas dentro de la organización educativa y, a la vez una semilla que genera nuevos cambios, nuevas transformaciones, para mejorar la calidad en todos los estamentos de una organización. (Carbonell, 2001; Estebaranz, 1994). 


\section{Metodología}

La investigación es de naturaleza cualitativa con un diseño biográfico-narrativo, el cual se emplea fundamentalmente en la educación para conocer la manera en que evoluciona la pedagogía, tomando como punto de partida las prácticas de los docentes en su quehacer cotidiano dentro del aula. Según Bolívar, Domingo y Fernández (2001) el enfoque biográfico narrativo permite conocer más profundamente lo que realmente sucede en el mundo escolar, a través de la mirada de los implicados, utilizando testimonios escritos donde narran sus experiencias, es decir con un alcance hermenéutico, narrativo, donde hacen escuchar su voz.

El alcance es descriptivo y se utilizan las técnicas de recolección de información cualitativas diario de campo y entrevista; se emplean listas de verificación. La población está constituida por los profesores de una institución educativa de Popayán, Cauca. La muestra se determinó mediante el muestreo no aleatorio por conveniencia y consta de tres profesores de secundaria. La construcción de los instrumentos se realizó a partir de las categorías: prácticas pedagógicas, innovación pedagógica y TIC, el cuestionario de entrevista consta de seis preguntas centrales y el análisis de información refiere a técnicas de codificación, así como metodologías para su análisis.

Saldańa (2013) expone que una de las formas de analizar la información cualitativa, es a través de la codificación, de manera que el empleo de un código adecuado es uno de los primeros pasos de la recolección de información, es vital; de hecho afirmar que:

Un código en una investigación cualitativa es frecuentemente una palabra o frase corta que de forma simbólica resume un atributo evocativo, con esencia sobresaliente de una parte de un texto o de un dato visual. La información puede ser la transcripción de una entrevista, las notas de campo, documentos, dibujos, fotografías, videos, sitios de Internet, literatura, etc. (p.3)

Miles y Huberman (2014), expresan que al ser los datos cualitativos «una fuente de descripciones ricas, bien fundamentadas y de explicaciones de los procesos humanos, preservan el flujo cronológico, permiten cuales eventos conducen a que consecuencias y derivar explicaciones útiles» (p. 1). Por tanto, su análisis permite que el investigador obtenga una visión holística de la realidad. Por esto se realizó una categorización axial.

\section{Resultados Y DisCUSIÓN}

A partir de la información obtenida en la entrevista realizada a la muestra, se identifican rasgos que indican el empleo de los recursos educativos digitales 
en la praxis educativa, en este apartado los profesores participantes serán mencionados con códigos que los identifican para conservar la confidencialidad de la investigación realizada.

\subsection{Identificación de características innovadoras en las prácticas pedagógicas mediadas por TIC}

Los últimos años han estado marcados por los cambios derivados de la inserción de las tecnologías en nuestras vidas, asistimos a una era digital, nuestra sociedad ha cambiado y esto sin duda ha tenido también un impacto en la educación y de manera directa en cómo aprendemos y cómo influyen los ambientes en los aprendizajes alcanzados. Desde este contexto las TIC se asumen como factor estratégico, hoy ante la crisis mundial derivada por la COVID-19, son el gran aliado de profesores y estudiantes que, ante la imposibilidad de volver a las aulas, han encontrado en las herramientas digitales una forma de acercarse a los estudiantes, compartir el conocimiento, generar nuevos entornos de enseńanza y aprendizaje, nuevas maneras de gestionar y transferir el conocimiento. Lo que ha demandado de manera especial un autoaprendizaje por parte de los profesores en competencias digitales.

Esta situación ha hecho que la mayoría de profesores enfrenten su miedo y resistencia al uso de las TIC en sus prácticas, y desarrollen habilidades y competencias hacia el diseño y creación de contenidos, apropiación y uso de recursos digitales, como lo plantea el Ministerio de Educación Nacional MEN (2013) al proponer como competencias a desarrollar por parte de los profesores las siguientes:

- Reconoce un amplio espectro de herramientas tecnológicas y algunas formas de integrarlas a la práctica educativa.

- Utiliza diversas herramientas tecnológicas en los procesos educativos, de acuerdo a su rol, área de formación, nivel y contexto en el que se desempeña.

- Aplica el conocimiento de una amplia variedad de tecnologías en el diseño de ambientes de aprendizaje innovadores y para plantear soluciones a problemas identificados en el contexto. (p. 36)

Lo que evidencia que la integración de las TIC a las prácticas requiere de un proceso de selección, planeación, desarrollo y evaluación de actividades y estrategias con mediación de TIC, para alcanzar los propósitos de aprendizaje, ya que como lo expresa Ortiz (2019) para que la innovación educativa aporte 
de manera significativa, es necesario establecer con claridad el uso de la tecnología, ya que vincularla a la práctica no garantiza procesos innovadores.

En virtud de los intereses de la investigación, desde los cuáles se esperaba conocer las características de innovación presentes en las prácticas mediadas por TIC, el análisis derivado de las entrevistas y el proceso de observación, permitieron identificar el uso de herramientas digitales como apoyo a los procesos de enseñanza y aprendizaje.

Como es el caso del profesor P1., profesor de matemáticas en los grados de básica secundaria, quién afirma que ha utilizado el software educativo Caja de polinomios, que es un simulador para las operaciones básicas con polinomios y las técnicas de factorización, así como también las temáticas de ecuaciones lineales, parámetros y áreas; esta es una estrategia lúdica, que permite que los profesores, utilicen las TIC para enseńar a sus estudiantes las matemáticas. La aplicación es en línea y se puede adquirir en el portal del MEN.

La profesora P2., dentro de su área de las ciencias sociales, también utiliza herramientas digitales en línea para fortalecer sus contenidos de enseñanza, utilizando los recursos que ofrece la página www.ciudadaniadigital.gov.co. De esta manera, permite que la tecnología se convierte en apoyo y estrategia didáctica para exponer hechos de la realidad social que susciten reflexiones individuales y grupales, para crear conciencia sobre los derechos de los ciudadanos, que son los constituyentes primarios de la sociedad, para conocer los mecanismos de protección de esos derechos en los órganos constitucionales existentes, pero también para motivar el respeto por los derechos de los demás y reconocer que existe igualmente deberes que cumplir asociados con cada uno de sus derechos.

Asimismo, P3., posee igualmente competencias digitales en la aplicación de las TIC en la educación desde su área profesional de ciencias sociales, introduce temáticas como la historia de las grandes civilizaciones de la humanidad, particularmente el hecho que estas florecieron alrededor de grandes ríos que fueron canalizados, exponiendo las características particulares de cada civilización mediante videos educativos.

De lo anterior, se puede reconocer que la innovación emerge en el acto educativo, como una acción de cambio con respecto a las formas tradicionales de comunicar el conocimiento por parte de los profesores y por esto la innovación, se configura a partir de estrategias pedagógicas y didácticas a través de las cuales los profesores representan, comunican y comparten los conocimientos. Desde esta perspectiva se puede plantear que la innovación es incremental, que si bien son innovaciones que no representan gran novedad, sí efectúan de manera significativa cambios en las prácticas de los profesores y contribuyen 
al desarrollo de los aprendizajes en los estudiantes y sobre todo son prácticas nuevas en el contexto específico en el que se desarrollan, puesto que, bajo el contexto de una institución educativa rural que presenta dificultades de conexión, se convierten en acciones valiosas e innovadoras que permiten resignificar las prácticas pedagógicas.

\subsection{Herramientas digitales utilizadas}

$\mathrm{Al}$ indagarse sobre las herramientas digitales que utilizan los profesores, P1., manifiesta que:

Tomi 7, que es un dispositivo que facilita el proceso de aprendizaje en el aula de clase y favorece la evaluación de la práctica del profesor, puesto que permite analizar si la metodología utilizada es buena o debe mejorarse, o se requiere hacer cambios que garanticen un mejor rendimiento en los estudiantes.

P2., replica que, «educaplay, youtube, son las aplicaciones que más utilizo en mis clases», y P3., añade que, "en mi práctica utilizo las redes sociales y el correo electrónico, tablet, computadores para educar con aplicaciones ya diseñadas». Además, se puede percibir que otras herramientas de la web 2.0, como los blogs o las wikis, no son muy utilizadas, ante lo cual los profesores expresan que los estudiantes no las acogen y la razón es que, como dice P3., «se han utilizado blogs donde las estudiantes suben sus opiniones sobre temas en particular, pero hubo poca participación porque a la mayoría de las estudiantes les cuesta escribir y publicar sus ideas».

De lo anterior se puede identificar que las TIC se emplean en el aula de clase, se utilizan en las ocasiones que se requiere, en función de las limitaciones de conectividad existentes, pero en función de los objetivos pedagógicos de las asignaturas. P1, manifiesta que «En el desarrollo de la clase, y la evaluación es interesante implementarla con Tomi 7 , aunque han sido muy pocas las veces, debido al problema de baja conectividad que se presenta en la institución». El profesor identificado como P2. añade que:

En el desarrollo de la clase y para la evaluación sería ideal vincular más herramientas digitales, pero la institución no tiene buena conectividad y en ocasiones las salas de internet se destinan para la clase de Informática y se dificulta implementar espacios para las otras áreas.

Estos relatos permiten reconocer las acciones realizadas por los profesores que, en virtud del contexto de la institución, aportan al fomento de procesos innovadores, ya que como lo plantea Rivas (2010), «la innovación 
es la introducción de algo nuevo dentro de una realidad existente» (p. 20), por lo anterior, se puede expresar que estas prácticas, han aportado de manera significativa a la generación de nuevos ambientes de aprendizaje y en coherencia buscan fomentar el desarrollo de habilidades y competencias en los estudiantes, que les permitan alcanzar los aprendizajes esperados.

Para hablar de innovación, es necesario reconocer el contexto en el que emergen las nuevas acciones de cambio, puesto que, no es lo mismo identificar acciones innovadoras, en instituciones que cuentan con los recursos y apoyo institucional para emprender cambios, a instituciones como la analizada en esta investigación, que carece de recursos tecnológicos y conectividad, en cuyo caso la innovación se reconoce desde una idea, instrumento, estrategia o proyecto pedagógico, aspectos que se hacen evidentes en estas prácticas, Valenzuela (2017) expresa que «el propósito de la innovación educativa es alterar la realidad modificando concepciones, actitudes, métodos e intervenciones, para así mejorar o transformar los procesos de enseñanza-aprendizaje» (p. 40).

Otro de los aspectos importantes que enfrentan los profesores al momento de realizar la aplicación práctica de las TIC en el aula se relaciona con los problemas de conectividad, asunto que es vital para la incorporación de herramientas digitales de la web 2.0 y 3.0. Esto ha impactado en la institución, para que no se tengan prácticas extracurriculares con el empleo de las TIC, es decir, los ambientes blended learning fuera del aula no se utilizan, por lo tanto, no existen las posibilidades para actividades tendientes al acompañamiento, seguimiento y evaluación del aprendizaje a través de estas plataformas. Esto se evidencia cuando P1., P2., y P3., responden que «No» cuando se les indaga si las TIC se aplican fuera del aula y cuáles son las estrategias para ello.

\subsection{Características de innovación en las prácticas pedagógicas}

$\mathrm{Al}$ indagar a los profesores acerca de las características de su práctica con el uso de las TIC, el grado de innovación que tienen en ellas y las estrategias utilizadas, se identificó a través de las entrevistas y en relatos como el del profesor P1. que, desde su perspectiva de aplicación en matemáticas, sostiene que: «básicamente en matemáticas no hay secreto, creo que una buena práctica debe ir acompañada de una buena explicación, y la utilización de las TIC hace que se vea más agradable y al maestro le facilita el trabajo». P2., expresa que:

en ciencias sociales, creo que después de una explicación, utilizar un video o una actividad interactiva, mediada por las TIC, hace que se afiance el conocimiento y sea agradable para el estudiante y para el profesor, ya que se facilita el trabajo. 
Y finalmente, P3., dice que:

Yo procuro obedecer a los estilos de aprendizaje de las estudiantes, digo procuro porque generalmente uno debe cumplir con un plan de trabajo. Pero sí, la estrategia se puede cambiar. Debido a las diferencias que hay en el aprendizaje de los estudiantes, yo les digo a ellas que utilicen las herramientas digitales que deseen, de acuerdo a las necesidades o capacidades que tenga cada una para el uso del equipo, o para ver el vídeo, según la disposición y el interés que tengan. En ese sentido soy muy flexible, pero siempre debe ir bien orientado porque se debe cumplir con un plan de trabajo.

Este mismo profesor destaca que su práctica es contextualizada y acorde a las necesidades evidenciadas por las estudiantes, así:

siempre tengo en cuenta la necesidad de las estudiantes para llegar a ellas. Me gusta identificar cuáles son las mejores prácticas y estrategias para hacerme entender dentro del aula y el desafio de siempre lograr la atención de ellas en un mundo que está reinado por las redes sociales y son un fuerte distractor, por tanto, lograr la atención de ellas es el reto más grande. En conclusión, atender la diversidad y lograr que ellas se involucren y aporten dentro del aula y que no solamente sea el profesor quien diga u oriente las cosas, sino que ellas también se involucren dentro del proceso.

Esto es un aspecto relevante dentro de los procesos formativos con uso de las TIC, puesto que hoy reconocemos el valor que estas tienen en los procesos de aprendizaje, siempre y cuando su uso considere aspectos como: propósitos de aprendizaje, necesidades educativas y recursos tecnológicos, con los cuales se busca potenciar aprendizajes a través de escenarios que le permitan a los estudiantes crear sus conocimientos, Gutiérrez, Gil, Zapata, Parra y Cardona, 2018, plantean:

Los procesos formativos modifican el rol del estudiante convirtiéndolo en sujeto activo del aprendizaje, y al profesor le da el carácter de guía y orientador en el intercambio comunicativo, para ayudar a resolver preguntas, dudas y posibilidades en el acceso a recursos, materiales de estudio y variedad de formas de comunicación entre los participantes. (p. 26)

De lo anterior, se reconoce el interés de los profesores por diversificar y contribuir a la mejora de su proceso de enseńanza, sin embargo a pesar de los esfuerzos realizados y de la capacitación de los profesores en el empleo de las tecnologías digitales dentro del aula y fuera de ella, existen limitaciones importantes como son la conectividad, la inexistencia o deficiencia de recursos tecnológicos, la falta de políticas de gestión tecnológica, dentro de los planes 
de gestión educativa, la deficiente promoción de la innovación en las prácticas pedagógicas; esto conlleva a que las prácticas pedagógicas innovadoras dentro del aula mediadas por TIC sean escasas.

Cómo ya se indicó, los desarrollos innovadores dependen en gran medida del impacto que generan al interior de la institución educativa y que este impacto está dado por las condiciones propias de la institución, en la figura 1 se presentan los principales aspectos identificados en las prácticas pedagógicas de los profesores y que aportan al desarrollo de procesos innovadores en el aula.

Figura 1. Características de la practicas pedagógicas innovadoras

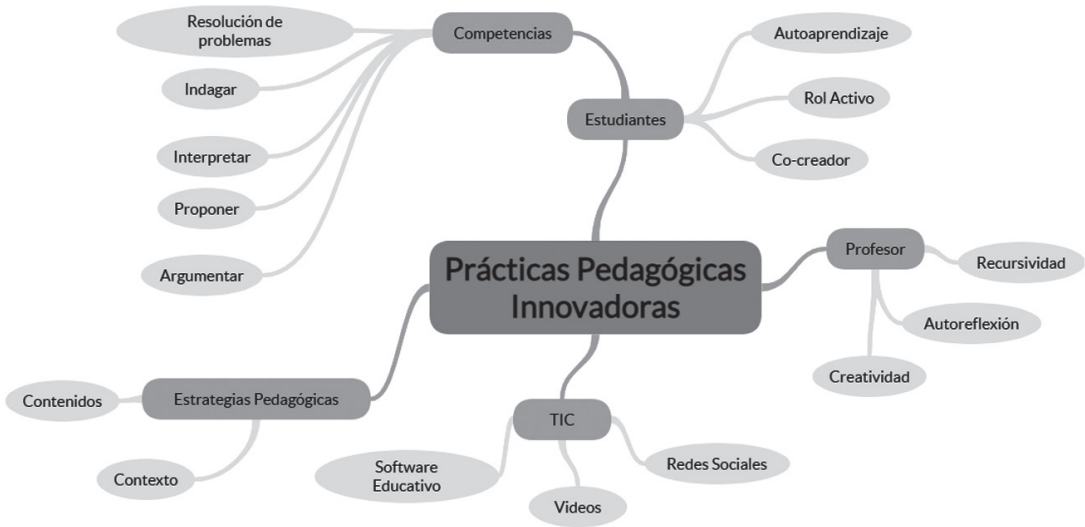

Fuente. Elaboración propia. Resultados de la investigación.

Por otra parte, la valoración de las prácticas pedagógicas con mayor nivel de innovación, se realizó siguiendo la valoración expuesta por González y Rojas (2007), donde se exponen criterios específicos para valorar las prácticas educativas, particularmente desde la innovación, en el instrumento se establecen los niveles de calificación (alto, medio y bajo), se asigna a cada criterio un puntaje entre 0 y 100 , de acuerdo con los siguientes rangos:

Bajo: $\mathrm{x}<=30$

Medio: $30<\mathrm{x}<=70$

Alto: $70<\mathrm{x}<=100$

Se calcula el puntaje total de la práctica, sumando los puntajes de todos los criterios de igual manera se establece el promedio, dividiendo el puntaje total entre el número de criterios y así se obtiene un número entre 0 y 100 , que permite establecer el nivel de desarrollo de la práctica pedagógica. En la tabla 1 se presentan estos resultados. 
Tabla 1. Valoración de prácticas pedagógicas

\begin{tabular}{|c|c|c|c|c|c|c|c|c|c|}
\hline \multirow[b]{2}{*}{ Criterios } & \multicolumn{3}{|c|}{ Profesor 1} & \multicolumn{3}{|c|}{ Profesor 2} & \multicolumn{3}{|c|}{ Profesor 3} \\
\hline & Alto & Medio & Bajo & Alto & Medio & Bajo & Alto & Medio & Bajo \\
\hline Fundamentación & 85 & & & 80 & & & 80 & & \\
\hline Pertinencia & 80 & & & 80 & & & 75 & & \\
\hline Consistencia & & 65 & & & 60 & & & 65 & \\
\hline Madurez & 90 & & & 85 & & & 85 & & \\
\hline $\begin{array}{l}\text { Empoderamiento } \\
\text { de actores }\end{array}$ & & 60 & & & 60 & & & 60 & \\
\hline $\begin{array}{l}\text { Evaluación y } \\
\text { seguimiento }\end{array}$ & 75 & & & & 65 & & 80 & & \\
\hline $\begin{array}{l}\text { Resultados e } \\
\text { impactos }\end{array}$ & 85 & & & & 65 & & 85 & & \\
\hline $\begin{array}{l}\text { Replicabilidad y } \\
\text { transferibilidad }\end{array}$ & 80 & & & 80 & & & 80 & & \\
\hline Sostenibilidad & 80 & & & 80 & & & 80 & & \\
\hline Innovación & 85 & & & & 60 & & & 60 & \\
\hline Total & & 785 & & & 715 & & & 750 & \\
\hline Promedio & & 78.5 & & & 71.5 & & & 75 & \\
\hline
\end{tabular}

Fuente: Elaboración propia. Resultados de la investigación.

Para describir las prácticas pedagógicas mediadas por TIC, utilizadas por los docentes de la muestra, se puede establecer el grado de innovación en ellas, analizando la forma en que estas responden a las competencias que se despliegan, las estrategias que se utilizan, las secuencias desarrolladas dentro del aula de clase y los productos obtenidos, así como el tipo de innovación que se ha introducido en dichas prácticas.

Las prácticas pedagógicas se orientan siempre a problemas en el contexto de los estudiantes, con el fin de generar soluciones de aprendizaje que fomentan competencias, habilidades y destrezas en función de la disciplina de estudio. Esto refleja ideas constructivistas en lo relacionado con la didáctica, donde el estudiante es parte activa del proceso de aprendizaje y los contenidos están en función de su realidad cotidiana. Sin embargo, se debe puntualizar que las competencias, destrezas y habilidades adquiridas en estas prácticas requieren coordinarse para que haya transversalidad e integración con otras áreas de estudio. En particular, la adquisición de destrezas y competencias que puedan transformar su conocimiento tácito, cotidiano en una práctica innovadora, 
es muy relevante, porque si esto no sucede, se cae en la rutina, en las formas sin autorreflexión del proceso de enseñanza y esto puede llevar a la ineficiencia de las prácticas pedagógicas utilizadas.

En cuanto a las herramientas para trabajar, siempre se utilizan las tecnologías que promueven la alfabetización digital ligada a praxis comunitarias, sociales, de ciudadanía participativa, hechas posibles por las nuevas opciones de gobierno abierto y e-Government que el Estado promueve desde las tendencias de una nueva gestión pública, siguiendo los lineamientos globales de la sociedad de la información. Sin embargo, en concreto, las herramientas de alfabetización digital e informacional por sí mismas no se emplean para construir competencias digitales en pro de la autonomía y la libertad.

En el área de matemáticas se emplean estrategias pedagógicas de corte propositivo y didáctico enmarcados en conceptos fundamentales de las matemáticas. A través de materiales visuales, ejercicios participativos, comunitario y propositivos, se incita a la participación activa de todas las estudiantes a través de preguntas, indagación, juegos y resolución de conflictos. Fortalecen las competencias interpretativas, propositivas y argumentativas de las estudiantes.

En el área de ciencias sociales se utilizan estrategias pedagógicas de corte humanista enmarcadas en conceptos fundamentales de las ciencias sociales y el eje educativo para la defensa y promoción de los derechos humanos en la construcción de paz. Además de indagar por conceptos previos y reforzarlos a través de videos educativos que profundizan los conocimientos y promueven la participación de las estudiantes de manera individual y colaborativa.

Otro aspecto relevante a evidenciar en los casos analizados es el asociado a los resultados de aprendizaje alcanzados por los estudiantes, ante lo cual el profesor P1. expresa que la vinculación de las TIC en sus prácticas «ha contribuido a la motivación e interés por el área y sí posibilitan la generación de ambientes de aprendizaje innovadores, lo cual se ha visto reflejado en un mejor rendimiento académico de los estudiantes», de manera similar plantea P2. que, con el uso de las tecnologías,

se ha logrado fortalecer el interés y motivación por aprender y han permitido que las estudiantes tengan experiencias agradables y significativas para facilitar su aprendizaje. Sin embargo, falta crear espacios para lograr utilizar de manera más efectiva lo que tenemos de recursos tecnológicos.

Este mismo profesor plantea que en su caso «las TIC son tenidas en cuenta para reforzar o motivar al estudiante frente a un tema como material de apoyo para hacer mejor el proceso educativo, aportando de manera significativa al cumplimiento de los objetivos de la materia». 
Para establecer los tipos de innovación utilizados en sus prácticas pedagógicas por los profesores, en esta investigación se utiliza la clasificación planteada en la denominada i-escala, que López y Heredia (2017) definen como «una herramienta enfocada en la evaluación de los proyectos de innovación educativa, el impacto que estos promueven en el proceso de enseñanza y aprendizaje, y la orientación del esfuerzo hacia acciones concretas en una transformación de mejora constante» (p. 5). La clasificación del tipo de innovación consta de una escala con tres categorías: disruptiva; revolucionaria; incremental.

De acuerdo con López et al., (2017), la innovación disruptiva «tiene capacidad y potencial para afectar a todo y a todos los que actúan en el contexto educativo; rompe la linealidad de un método, técnica o proceso de enseñanza y aprendizaje y la transforma drásticamente» (p. 19). La innovación revolucionaria se genera al aplicar «un nuevo paradigma que cambia radicalmente el proceso enseñanza y aprendizaje, modificando significativamente las prácticas existentes» (p. 19). Por ello se denomina también innovación radical.

Asimismo López et al. (2017) conceptualizan la innovación incremental como "un cambio que se construye con base en los componentes de una estructura ya existente, dentro de una arquitectura o diseño establecido" (p. 19). Es un proceso en el que se da a conocer, de forma incremental, la manera en que se puede utilizar un nuevo elemento en el contexto educativo.

En este orden de ideas, se puede afirmar que, tanto en el área de matemáticas como de ciencias sociales, el tipo de innovación que se encuentra es incremental, articuladas las actividades de innovación dentro del marco metodológico y diseńo existentes, de manera que el empleo de las TIC es paulatino, no solo por los estilos de aprendizaje de los profesores y de los estudiantes, sino también por los problemas de conectividad dentro de la institución educativa, que impiden el intercambio de experiencias.

Es rescatable la labor del docente P1., en el área de matemáticas, por ello, es necesario fomentar una cultura de la calidad y de la innovación al interior de las instituciones educativas, que permitan visibilizar y comunicar las experiencias innovadoras que están realizando los profesores y que aportan al desarrollo de los procesos académicos de la institución. Al respecto, Parra y Agudelo (2020), plantean que «la innovación educativa es un proceso que genera una transformación a nivel organizacional, administrativo y pedagógico, en busca de la mejora continua de los procesos de enseńanza y aprendizaje» (p. 12), en este sentido, la innovación se convierte en un compromiso de todos, surge desde una necesidad de cambio evidenciada en la práctica pedagógica, pero sí esta iniciativa se convierte en un trabajo colegiado y participativo, sin duda tendrá mayor impacto en la institución y podrá contribuir al enriquecimiento de otras prácticas. 


\section{Conclusiones}

Los profesores desarrollan prácticas pedagógicas innovadoras, de tipo incremental, porque están vinculando acciones paulatinas, de acuerdo a las necesidades que se presentan en el aula, y estas innovaciones pueden posteriormente darse a conocer para que otros profesores las apliquen; este tipo de innovación permite realizar cambios sobre las prácticas existentes, mejorándolas, pero no alcanzan un nivel de impacto o transformación que vaya más allá del aula.

Los profesores se han capacitado en el empleo de las TIC en el aula, siguiendo los lineamientos del MEN y de MinTIC (Ministerio de las tecnologías de la información y la comunicación), las aplican en función de su experiencia y necesidades, pero no de forma más continua, ya que no existe una política de gestión educativa orientada a fomentar acciones innovadoras.

En las prácticas pedagógicas de los profesores se observa el ciclo de la calidad, es decir, se orientan los procesos hacia la mejora continua de sus prácticas, pero también un esquema riguroso, con poca flexibilidad, que no permite que el conocimiento tácito se convierta en oportunidad de conocimiento crítico e innovador, que pueda incrustarse dentro del paradigma tecno-económico de la actual sociedad de la información y el conocimiento.

Las herramientas web 2.0 utilizadas por el profesor de matemáticas incluyen tomi 7, software promovido por el MEN; en el área de sociales se utiliza los recursos de la plataforma InnovaTIC, y a veces los blogs, aunque estos, según la apreciación de una de las profesoras, no es muy acogida por los estudiantes debido a que no les gusta leer.

Las prácticas de los profesores pueden catalogarse como buenas prácticas, en el criterio de alto cumplimiento según la guía 28 del MEN para las buenas prácticas, sin embargo, el grado de innovación en ellas está en dos casos por debajo de lo requerido.

Se deben apoyar las prácticas pedagógicas innovadoras mediadas con TIC mucho más, como lo plantea Baracaldo (2019), al indicar que los profesores deben identificar en la planeación de sus cursos la posibilidad de vincular las tecnologías e identificar los problemas de acceso, para lo cual es pertinente que desde las instituciones educativas se fortalezca, la planeación estratégica enfatizando en estrategias pedagógicas apoyadas en el blended learning, en pruebas de medición de estilos de aprendizaje, en equipamiento tecnológico y conectividad adecuada, así como en trabajo en equipo con la comunidad educativa, 


\section{REFERENCIAS BIBLIOGRÁFICAS}

Baracaldo, D. (2019). Technology Integration for the Professional Development of English Teachers. Tecné, Episteme y Didaxis: TED, (46). https://doi. org/10.17227/ted.num46-10545

Bolívar, A., Domingo, J. y Fernández, M. (2001). La investigación biográficonarrativa en educación. Enfoque y metodología. Madrid: La Muralla.

Carballo, R. (2006). Innovación y gestión del conocimiento. Madrid: Ediciones Díaz de Santos.

Carbonell, J. (2001). La aventura de innovar. El cambio en la escuela. En Razones y propuestas educativas. Madrid: Ediciones Morata.

Estebaranz, A. (1994). Didáctica e innovación curricular. Sevilla: Universidad de Sevilla

Fullan, M. y Stiegelbauer, S. (1991). The New Meaning of Educational Change. Londres: Casell.

González, L., y Rojas, C. (2007). Aprendizajes para mejorar. Guía para la gestión de buenas prácticas, 29. MEN. Recuperado de https://www.mineducacion. gov.co/1621/articles-124660_archivo_pdf.pdf

Gutiérrez, M., Gil, H., Zapata, M., Parra, L. y Cardona, C. (2018). Uso de las herramientas digitales en la enseñanza y aprendizaje universitario. Una propuesta pendiente por construir. Manizales: Universidad Católica de Manizales.

López, C. y Heredia, Y. (2017). Marco de referencia para la evaluación de proyectos de innovación educativa: Escala i. Monterrey: Instituto Tecnológico y de Estudios Superiores de Monterrey.

Miles, M. y Huberman, A. (2014). Análisis de datos cualitativos (3rd ed.). SAGE.

Ministerio de Educación Nacional - MEN (2013). Las competencias TIC para el desarrollo profesional docente. Recuperado de: https://www.mineducacion. gov.co/1759/articles-339097_archivo_pdf_competencias_tic.pdf

Ortiz, F. (2019). Los retos de las innovaciones educativas hoy: los docentes, las escuelas y los centros de innovación. Revista Educación y Ciudad, 2(37), 91-101. https://doi.org/10.36737/01230425.v2.n37.2019.2150

Parra, L. y Agudelo, A. (2020). Innovación en las prácticas pedagógicas mediadas por TIC. En R. Canales y C. Herrera (eds.). Acceso, democracia y comunidades virtuales (pp. 51-64). Buenos Aires: CLACSO. https://doi. org/10.2307/j.ctv1gm00v8.6

Pila, J. C., Andagoya, W. y Fuertes, M. (2020). El profesorado: Un factor clave en la innovación educativa. Revista EDUCARE - UPEL-IPB - Segunda Nueva Etapa 2.0, 24(2), 212-232. https://doi.org/10.46498/reduipb.v24i2.1327 
Rivas, A. (2017). Cambio e innovación educativa: las cuestiones cruciales. Buenos Aires: Santillana.

Rivas, M. (2010). Innovación educativa. Teoría, procesos y estrategias. España: Editorial Síntesis, S.A.

Saldaña, J. (2013). Manual de Codificación para investigadores cualitativos (2nd ed.). SAGE.

Salinas, J. (2004). Innovación docente y uso de las TIC en la enseñanza universitaria. RUSC. Revista Universidades y Sociedad del Conocimiento, 1 (1), 1-16. [Fecha de consulta 6 de abril de 2021]. Recuperado de https://www. redalyc.org/articulo.oa?id=78011256001

Salinas, J. (2008). Innovación educativa y uso de las TIC. Sevilla: Universidad Internacional de Andalucia.

Unesco. (2016). Innovación educativa. París: Unesco.

Unesco. (2018a). Construyendo las destrezas digitales del mañana. Recuperado de http://unesdoc.unesco.org/images/0026/002618/261853e.pdf

Unesco. (2018b). Destrezas para un mundo conectado. París: Unesco.

Valenzuela, J. (2017). La innovación como objeto de investigación en educación: problemas, tensiones y experiencias. En M. Ramírez y R. Valenzuela (eds.), Innovación educativa. Investigación, formación y visibilidad (pp. 29-50). Madrid: Editorial Síntesis S. A. 